\title{
Divide et Impera: Efficient Synthesis of Cyber-Physical System Architectures from Formal Contracts
}

\author{
César A. R. dos Santos ${ }^{1,2}$, Tom Schrijvers ${ }^{2}$, Amr H. Saleh ${ }^{1}$, and Mike Nicolai ${ }^{1}$ \\ 1 Siemens Digial Industries Software \\ 2 KU Leuven
}

\begin{abstract}
Generative Engineering is a new paradigm for the development of cyber-physical systems. Rather than developing a single, increasingly more detailed model of a system, multiple architectural variants are computationally generated and evaluated, which would be prohibitively expensive to do by hand. Existing synthesis approaches are geared towards finding one solution fast, but this makes them less effective for generative engineering where we are interested in enumerating many or all solutions. The common approach in generative engineering is to compute a new verification problem per generated architecture, despite all being variants of the same verification problem. This makes the tools unable to exploit commonalities and they end up doing much of the same verification work over and over again.

Our work addresses this inefficiency in the synthesis of all correct-by-construction logical architectures of a system with a simple but effective approach. We create only one parameterized verification problem per use case, and, by exploiting the assumption mechanism of SMT solvers, we can very efficiently and incrementally check each generated architecture.

Our experimental evaluation demonstrates that this approach is orders of magnitude faster than the typical synthesis approach.
\end{abstract}

Keywords: Generative Engineering, Contract-Based Design, Cyber-Physical Systems

\section{Introduction}

Many industrial systems are succumbing under the weight of their own complexity. Innovation is becoming increasingly risky, and architectural innovation in particular has actually decreased over time [11].

Architecture, in the context of this paper, refers to the set of components and the connections between those components that form a particular implementation of the system. The system architecture [12] is defined early in the design process, and its formulation is often a manual process with limited feedback. Feedback on the quality of the architecture only arrives later, once detailed models are available.

If the architecture turns out to be suboptimal, or even infeasible, then a redesign is necessary. Not only does the system architecture have to be changed, but also all the models that are derived from it. Such redesign cycles are expensive, so architectures tend to closely follow older designs that were proven to work [11].

To allow for architectural innovation, engineers need methodologies and tools that allow them to efficiently explore many candidate designs early in the design process. 
Accomplishing this is the goal of the computational design synthesis field (a.k.a. Generative Engineering), where tools like Siemens Simcenter Studio [2] computationally synthesize all correct-by-construction architectures directly from a system model.

One major issue with existing approaches is that they are inefficient when it comes to enumerating all solutions: they translate each architecture to a separate variant of the same verification problem. This leads to a lot of redundant verification work and prevents the exploitation of commonalities. Architectures can be generated extremely efficiently with the right encoding, so we want to minimize as much as possible the cost of verification. The more architectures to explore, the cheaper verification needs to be.

Contributions The goal of this work is to develop a synthesis methodology, targeted at generative engineering, that enumerates all valid architectures of a system as efficiently as possible. We accomplish this through the following contributions:

1. We introduce a methodology that efficiently synthesizes all correct-by-construction architectures of a system, from formal contracts written in Linear Temporal Logic (LTL) [6] with arithmetic, through the incremental application of a single parametric encoding of the verification problem per use case.

2. We evaluate our methodology on three use cases, and show that it is orders of magnitude faster than the naive synthesis approach.

3. We also evaluate our methodology on a range of solvers, and contrast the efficiency of our approach with the solvers' internal $\exists \forall$ reasoning, and show that our approach is faster as well.

\section{Background}

Before explaining our methodology, it is important to first understand the typical industrial design process. While the steps may vary between companies, they can be summarized into 4 phases: requirements, functions, logical and physical. To illustrate that process, we use a Redundant Sensor example adapted from OCRA [1].

The first phase of the design process focuses on requirement gathering. We assume that requirements are translated into formal specifications by an expert.

After gathering requirements, the next step is to figure out which functions the system must implement and to map those to the components which form the system implementation. The outcome is a hierarchical system model, as depicted in Fig. 1.

In this case the system is divided into two Sensors, which measure the environment; a Selector component, which handles error correction in the presence of failures; and two different types of monitors (a Variance Monitor and a Difference Monitor) which attempt to detect sensor failures based on the temporal variance and absolute difference of the sensor outputs, respectively. All the monitors have a cardinality $[n: m]$ (lower and upper bound on the number of allowed instances) that is variable, because we do not know a priori how many we will need to achieve a functioning system.

Once the system model is defined, we create the logical architecture for the system. A logical architecture specifies in an abstract manner the set of components that constitute the system implementation and how they are connected. Fig. 2 shows one of the possible logical architectures of the system. 


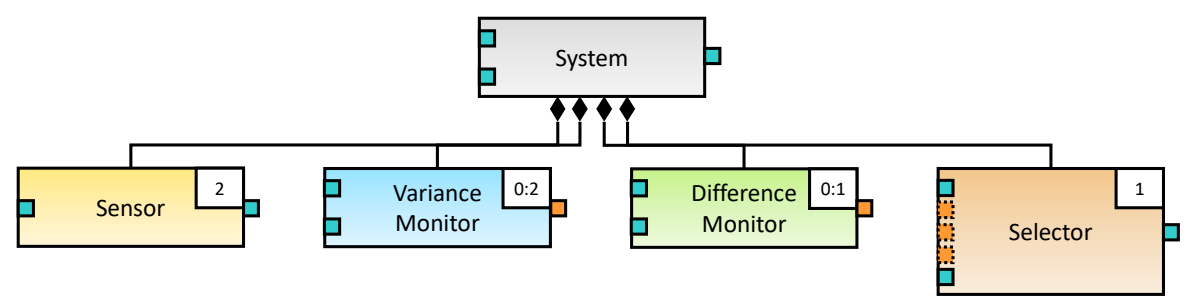

Fig. 1: The system model for the Redundant Sensors use case. The allowed number of instance per component is annotated in their top-right corners. Some ports of the selector have a dotted outline to indicate that they are optional.

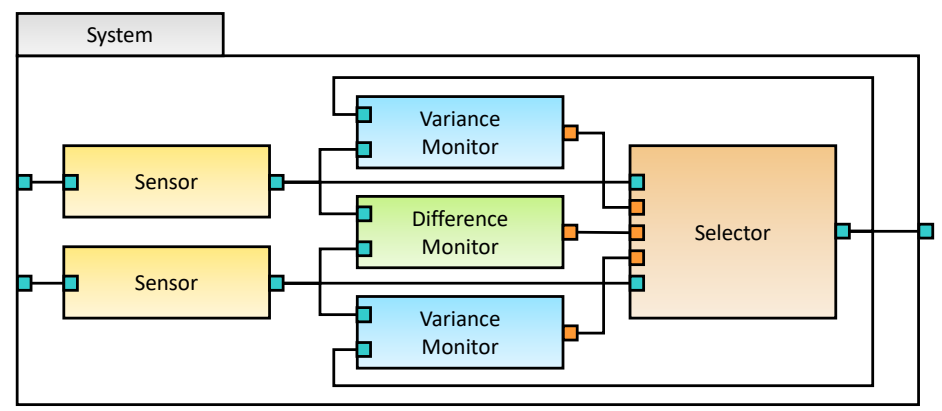

Fig. 2: One possible architecture for the Redundant Sensors use case. We want to know if there are others that also lead to working solutions, particularly with fewer monitors.

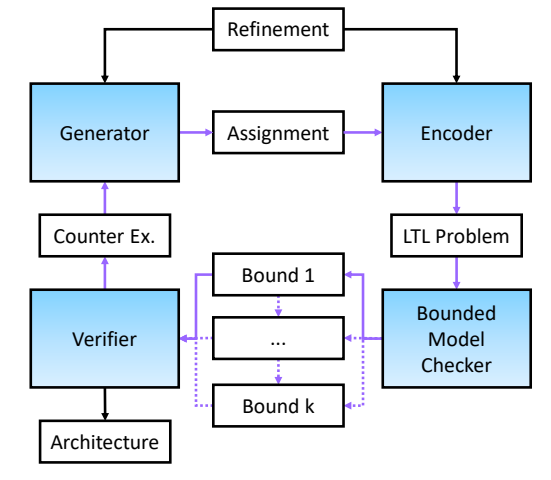

Fig. 3: The usual synthesis pipeline. The purple arrows indicate the synthesis loop per generated architecture.

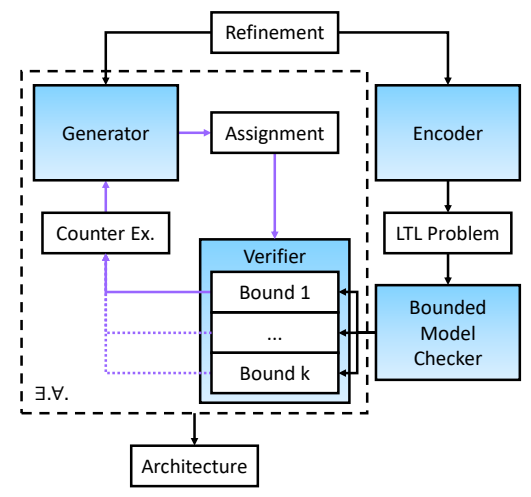

Fig. 4: Our synthesis pipeline, note the shorter loop. The generator and verifier can be merged with an $\exists \forall$ encoding.

With the logical architecture in place, the next phase is to create one or more detailed physical models of the system, e.g. simulation models. These allow the engineers to evaluate the quality of the architecture and redo the design if it is suboptimal or infeasible. 
Our methodology changes the last two steps. Instead of manually developing one or more logical architectures and corresponding models, we synthesize these automatically from the system model. In the next section we explain how this is accomplished.

\section{Methodology}

Our synthesis methodology is structured around a pipeline, shown in Fig. 4. The basic idea is adapted from Simcenter Studio al. [2] and an extension of previous work [15]. Fig. 3 shows the typical synthesis approach for contrast.

Our methodology starts from the system model, which defines which components are available, what their interfaces are, and how many of them may appear in the system. In our methodology, the problem is defined as a set of contracts, one for each component (including the system itself), and a refinement specification that defines the structure of the system model. See Fig. 5 for the contract of the Redundant Sensor example.

Each contract defines the interface of the component and one or more viewpoints, which are independent sets of requirements comprised of the assumptions the component makes about its environment and which guarantees it provides under those assumptions. The refinement specification and the associated contracts are encoded into a generation problem and a verification problem. These two problems are encoded only once per use case, and then enter a loop (marked in purple). The loop works as follows:

Generation Is there a set of components (an ensemble) and a set of connections that connects all the ports in that ensemble? If so, create that architecture and go to Verification. If not, terminate.

Verification Does that architecture lead to a counter-example to the contract refinement? If not, save it, add constraints to prevent the architecture from being generated again, and loop back to Generation. If it is a counter-example, discard the architecture, add additional constraints to block all architectures that would lead to the same counter-example, and loop back to Generation.

We use Counter-Example Guided Inductive Synthesis (CEGIS) to guide the search [3]. When the verifier produces a counter-example, we take the bound-unrolled verification formula and replace each attribute and port variable with their respective values in the counter-example. Because the formula is parametric, the only remaining free variables are the boolean component and connection variables. By adding a negation of this formula to the generator, no other assignment to the component and connection variables that would lead to the same assignment of the port and attribute variables (the counter-example) is allowed.

Alternatively, the Generation step and the Verification step can be merged into a single problem using an $\exists \forall$ encoding and an $\exists \forall$ capable solver. This allows requirements to directly guide the search without an external CEGIS loop. In the next section we explain how these two steps are encoded. 


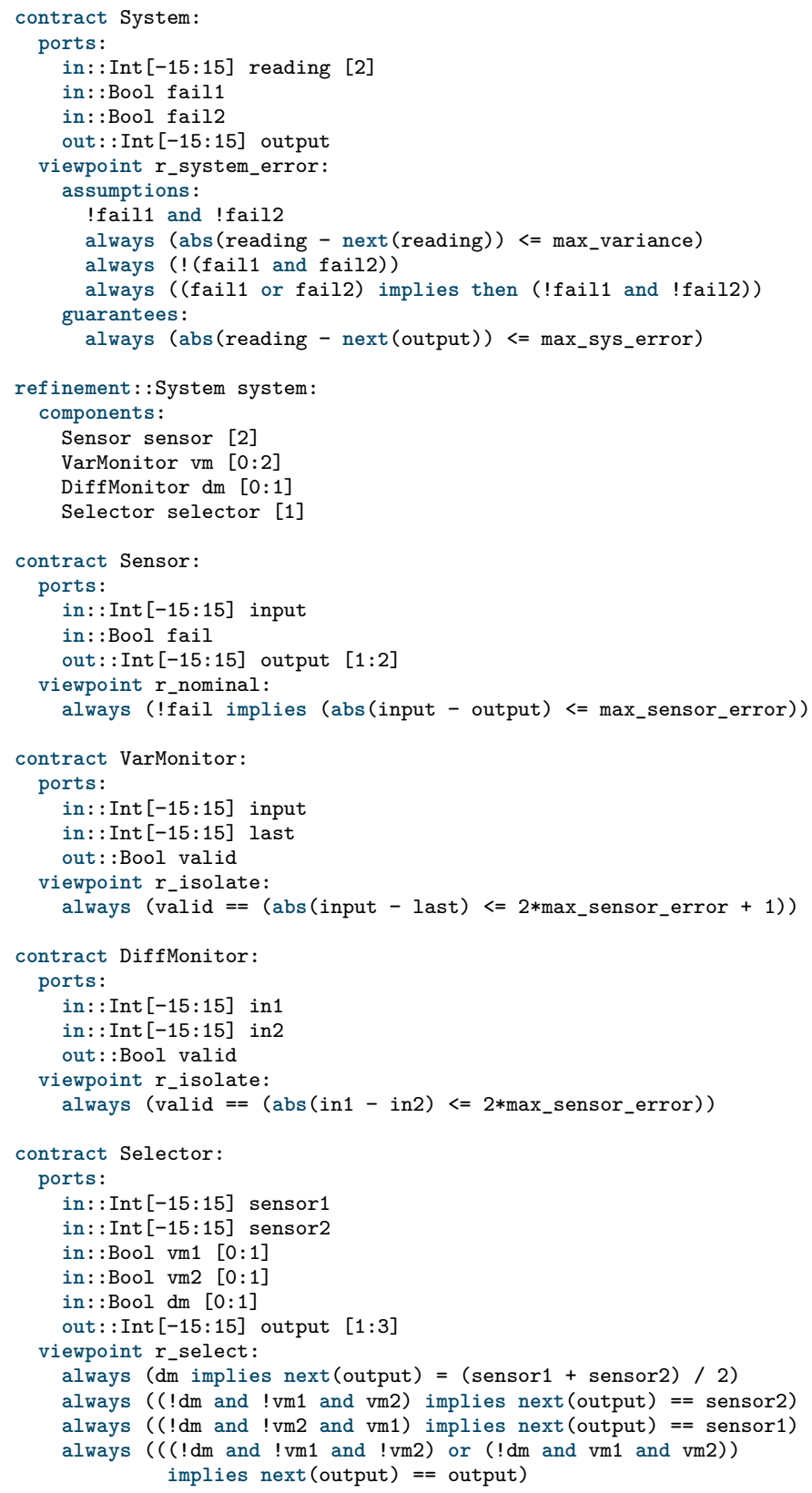

Fig. 5: The contracts for the Redundant Sensor use case. 


\section{Encodings}

Generation The goal of Generation is to compute sets of component instances and sets of connections between ports of those instances. The encoding for Generation is shown below:

$$
\begin{aligned}
& \text { for } c \in \mathbb{C} \text { : assert } \sum_{i=1}^{\lceil c\rceil} c_{i} \geq\lfloor c\rfloor \\
& \text { for } c \in \mathbb{C}, 1<i \leq\lceil c\rceil \text { : assert } c_{i} \rightarrow c_{i-1} \\
& \text { for } c . p \in \mathbb{P}, i \leq\lceil c\rceil \text { : assert }\lfloor c . p\rfloor \times c_{i} \leq \sum \mathbb{Q}_{c_{i} . p} \leq\lceil c \cdot p\rceil \times c_{i}
\end{aligned}
$$

We use $\mathbb{C}$ to refer to the set of all components in the system, $c$ to refer to a particular component, $\lfloor c\rfloor$ to refer to the lower cardinality of component $c$, and $\lceil c\rceil$ to refer to the upper cardinality of component $c$. To refer to a component instance, we use $c_{i}$, where $i \in \mathbb{N}$. The presence of component instances in the system is modeled as $\lceil c\rceil$ boolean variables for each $c$. Equation 1 constrains the number of component instances of each component to be within the bounds of its cardinality. Equation 2 enforces an ordering on the component instance variables.

We use $\mathbb{P}$ to refer to the set of all ports in the system, c.p to refer to a particular port, $\lfloor c . p\rfloor$ to refer to the lower cardinality of port c.p, and $\lceil c . p\rceil$ to refer to the upper cardinality of port c.p. $c_{i} . p$ refers to the port of a component instance $c_{i}$. We use $\mathbb{Q}$ to refer to the set of all possible connections between ports, and $\mathbb{Q}_{c_{i} . p}$ to the subset of possible connections involving port $c_{i} . p$. Each possible connection $a_{i} . x \triangleright b_{j} . y \in \mathbb{Q}$ is modeled as a boolean variable. A connection is possible if the two ports have opposite directions and the same type.

For each port $c_{i} . p$, we require that the number of times it connects to other ports is within the cardinality of $c . p$ and we multiply the cardinality by $c_{i}$ to ensure that the port cannot be connected when the corresponding instance is not present. Symmetry breaking constraints are elided for space reasons.

The output of Generation is an assignment to the instance and connection variables. Each assignment is passed as assumptions to Verification.

Verification The goal of Verification is to filter out ensembles or architectures that do not follow the system requirements. It is based on the contract refinement check ( $\preceq$ ) of the contract meta-theory of Benveniste et al. [5]. The encoding for Verification is shown below:

$$
\begin{aligned}
& \text { for } a_{i} . x \triangleright b_{j} . y \in \mathbb{Q} \text { : assert } a_{i} . x \triangleright b_{j} . y \rightarrow a_{i} . x=b_{j} . y \\
& \text { assert } A_{s} \wedge\left(\bigwedge c_{i} \rightarrow G_{c_{i}}\right) \rightarrow G_{s} \\
& c \in \mathbb{C} \backslash\{s\}, i \leq\lceil c\rceil \\
& \text { for } c \in \mathbb{C} \backslash\{s\}, i \leq\lceil c\rceil \text { : assert } A_{s} \wedge\left(\bigwedge o_{j} \rightarrow G_{o_{j}}\right) \rightarrow A_{c_{i}} \\
& o \in \mathbb{C} \backslash\{s, c\}, j \leq\lceil o\rceil
\end{aligned}
$$

We use $s$ to refer to the system instance, $A_{c_{i}}$ to refer to the assumptions of $c$ projected to instance $c_{i}$, and $G_{c_{i}}$ to refer to the guarantees. Each port instance $c_{i} . p$ is modeled as a variable of the type defined for port c.p. 
Equation 4 enforces that if a particular connection is present, the connected ports must have the same values. Equation 5 enforces that the composition of the component instances (according to their presence) alongside the system assumptions implies the system guarantees. Finally, Equation 6 enforces that for every component instance, its assumptions are implied by the assumptions of the system and the composition of all the other component instances.

Verification needs to hold for all possible states. It can be merged with Generation by encoding the problem in first-order logic, and using an $\exists \forall$ capable solver. This avenue was previously explored in CONDEnSe [15]. We support this merged configuration as well, but we found performance to be suboptimal. Instead, we encode the negation of the refinement constraints, and check for unsatisfiability, i.e. that the solver cannot find a counter-example. We use CEGIS [3] to add additional constraints to the Generation step as we verify each architecture, to prevent the generation architectures that would lead to the same counter-example.

We apply a time bounded, LTL satisfiability check with a user-defined bound $k$. Constraints are unrolled for all $i \in[1, k]$ once. Because the encoding is parametric and accepts the generated architectures as assumptions, we can check all unrollings very efficiently as they are encoded only once but executed many times.

\section{Evaluation}

Because other synthesis tools work very differently, a direct comparison is difficult, so we focus on evaluating the best way to synthesize with our encoding. We evaluate our work on 3 use cases: The running Redundant Sensors example, an XOR circuit built out of NAND gates, and a 4-bit adder circuit.

We contrast 3 different synthesis approaches: a) using an external verifier; b) using our one-time parametric encoding; and c) using an $\exists \forall$ encoding.

We run approach a) using only our fastest supported SMT solver for architecture generation and fastest supported LTL verifier (nuXmv [7]) configured for bounded model checking. For approaches b) and c) we use 3 different SMT solvers with $\exists \forall$ capabilities: Yices2 [9], Z3 [13] and CVC4 [4].

The following tables show the results for each use case and for each solver. The first row, Yices $2+$ nuXmv, shows the naive approach with an external verifier, generating a new problem each time and without CEGIS. The other rows show our approach (generate and verify, GV) on a variety of solvers, and the variant using $\exists \forall$ reasoning (EF). Unlike $\mathrm{Z} 3$ and CVC4, Yices 2 exposes its model generalization API, so we can take advantage of this to show how many architectures it explores in the EF case. GV (Yices2+) is a special case which combines our approach with Yices2's model generalization API.

Columns G. Archs and V. Archs are the number of generated and verified architectures, respectively. The total time includes the overhead of extracting the counterexample, processing it, and encoding it back to the generator, which can be substantial due to our Python implementation.

Our tests were executed on a laptop with a 4 core Intel i7-8650U CPU clocked at 2 $\mathrm{GHz}, 16 \mathrm{~GB}$ of RAM, running Ubuntu 20.04. Tests had a 1 hour time limit.

We can see from the results that our approach combined with the model extraction capabilities of Yices 2 gives the best results, computing all the solutions to all 3 problems 
Table 1: Comparison of different synthesis approaches for the Redundant Sensor use-case.

\begin{tabular}{lrrrrr}
\hline Approach & G. Archs & V. Archs & G. Time (s) & V. Time (s) & Total (s) \\
\hline Yices2+nuXmv & 13985 & 48 & 116.25 & 2664.55 & 2797.40 \\
GV (Yices2+) & 2977 & 48 & 1.73 & 6.05 & $\mathbf{8 . 5 9}$ \\
GV (Yices2) & 2980 & 48 & 2.01 & 8.37 & 54.94 \\
GV (Z3) & 4432 & 48 & 17.37 & 77.49 & 638.85 \\
GV (CVC4) & 3191 & 48 & 15.74 & 542.46 & 646.68 \\
EF (Yices2) & 4580 & 48 & 5.40 & 122.47 & 132.28 \\
EF (Z3) & 0 & 0 & 3600.0 & - & TIMEOUT \\
EF (CVC4) & 0 & 0 & 3600.0 & - & TIMEOUT \\
\hline
\end{tabular}

Table 2: Comparison of different synthesis approaches for a NAND-based XOR circuit

\begin{tabular}{lrrrrr}
\hline Approach & G. Archs & V. Archs & G. Time (s) & V. Time (s) & Total (s) \\
\hline Yices2+nuXmv & 7705 & 32 & 27.09 & 218.49 & 250.96 \\
GV (Yices2+) & 147 & 32 & 0.18 & 0.07 & $\mathbf{0 . 2 7}$ \\
GV (Yices2) & 141 & 32 & 0.19 & 0.10 & 0.77 \\
GV (Z3) & 142 & 32 & 0.45 & 0.69 & 5.09 \\
GV (CVC4) & 172 & 32 & 16.46 & 0.80 & 18.17 \\
EF (Yices2) & 509 & 32 & 0.43 & 0.32 & 0.87 \\
EF (Z3) & 32 & 32 & 27.29 & - & 27.37 \\
EF (CVC4) & 32 & 32 & 5.19 & - & 5.20 \\
\hline
\end{tabular}

Table 3: Comparison of different synthesis approaches for a 4bit adder circuit.

\begin{tabular}{lrrrrr}
\hline Approach & G. Archs & V. Archs & G. Time (s) & V. Time (s) & Total (s) \\
\hline Yices2+nuXmv & 43725 & 0 & 1465.91 & 2132.95 & TIMEOUT \\
GV (Yices2+) & 2333 & 16 & 5.24 & 5.27 & $\mathbf{1 1 . 1 1}$ \\
GV (Yices2) & 2280 & 16 & 6.66 & 7.31 & 51.53 \\
GV (Z3) & 1613 & 16 & 26.59 & 25.15 & 250.51 \\
GV (CVC4) & 304 & 16 & 274.81 & 119.31 & 400.37 \\
EF (Yices2) & 3837 & 16 & 8.18 & 13.31 & 24.14 \\
EF (Z3) & 0 & 0 & 3600.0 & - & TIMEOUT \\
EF (CVC4) & 16 & 16 & 59.85 & - & 59.91 \\
\hline
\end{tabular}

in a few seconds. The EF encodings using Z3 had very poor results, while CVC4 did well in the boolean circuit use-cases but timed-out in the Redundant Sensors use-case.

\section{Related Work}

There are many different methodologies and tools for synthesis, requirement verification and design exploration of hybrid systems and sub-problems thereof. It would not be feasible to do the entire field justice, so we chose to focus on the closest related works. 
Nuzzo et al. The work of Nuzzo et al. [14] was the main inspiration for our work, where the same contract meta-theory is applied to the synthesis of an electric power system (EPS). We follow their idea of splitting the problem into separate viewpoints, but Nuzzo et al. manually encode the generation and verification problems for their use case, while our approach is use-case agnostic.

Simcenter Studio Simcenter Studio [2] was the inspiration for our Generation encoding. Simcenter Studio focuses on the efficient but separate generation of ensembles and architectures, with the verification and evaluation of the candidates being entirely userdefined. This has the advantage of wide industry applicability. Engineers can specify and evaluate the requirements in the way that is most natural to their domain, but it precludes the requirements from participating in the search, leading to the generation of many spurious candidates.

Contract-Based Synthesis Tools Requirements in our approach are modeled in the contract meta-theory of Benveniste et al. [5], because it is highly modular and agnostic of the underlying logic of the contracts. There are many tools that support the same Assume/Guarantee style of contracts that we use, and three in particular also support synthesis: CONDEnSe [15], OCRA [8] and PyCo [10].

CONDEnSe was our previous attempt at a contract-based synthesis tool, but it lacked the features that were introduced in this work, namely: LTL support, component-level instantiation, CEGIS, and symmetry breaking.

OCRA is mainly a contract verification tool, and its input language served as one of the inspirations for ours. OCRA's 2.0 release added synthesis support, but it falls on the user to make their model parametric. OCRA does not handle the instantiation of connections itself which means there is no built-in symmetry breaking support and that it is possible to make the problem unbounded.

The PyCo tool of Iannopollo et al. [10] has some similarities to our approach in that it also focuses on synthesis of architectures from a library of components annotated with contracts. It uses very different encodings however, and focuses on finding a single, optimal architecture. As in our naive case, it generates a new verification problem for each candidate solution which it then sends to nuXmv, but with a CEGIS loop. As shown by our results, the cost of running nuXmv on each generated architecture is substantial. The tool also lacks support for arithmetic.

\section{Conclusions and Future Work}

In this paper we presented a synthesis methodology, targeted at generative engineering, that enumerates all valid architectures of a system as efficiently as possible. To do so, we adapted the general synthesis framework of Simcenter Studio to support contract-based verification, and use it to guide the search.

Our encodings allow us to efficiently verify each generated architecture on a single, unrolled refinement verification problem, leading to substantially better performance than the common synthesis approach. Our solution supports both CEGIS and $\exists \forall$ reasoning to guide the generation of architectures towards valid solutions, and we were surprised to 
find that the $\exists \forall$ support in existing SMT solvers was suboptimal for synthesis problems of this type, with Yices 2 being a standout exception, beating all other solvers.

There are two main areas of future work for this project: 1) more expressive specification logics, and 2) optimization.

Supporting more expressive logics is possible via various reductions to LTL.

When it comes to optimization, the naive solution is to restrict each new solution to be better in some metric than the last valid solution until no more solutions exist. It is worthwhile exploring if there is a better approach, taking advantage of a solver with direct optimization support. One major challenge is how to handle objectives that require simulation to evaluate (e.g. fuel consumption).

\section{References}

1. OCRA examples. https://ocra.fbk.eu/pmwiki.php?n=Main.Examples (2021), accessed: 2021-05-05

2. Simcenter Studio. https://www.plm.automation.siemens.com/global/en/ products/simcenter/simcenter-studio.html (2021), accessed: 2021-08-03

3. Abate, A., David, C., Kesseli, P., Kroening, D., Polgreen, E.: Counterexample Guided Inductive Synthesis Modulo Theories. In: Computer Aided Verification. pp. 270-288. Lecture Notes in Computer Science (2018)

4. Barrett, C.W., Conway, C.L., Deters, M., Hadarean, L., Jovanovic, D., King, T., Reynolds, A., Tinelli, C.: CVC4. In: Computer Aided Verification - 23rd International Conference, CAV 2011, Snowbird, UT, USA, July 14-20, 2011. Proceedings. Lecture Notes in Computer Science, vol. 6806, pp. 171-177 (2011)

5. Benveniste, A., Caillaud, B., Nickovic, D., Passerone, R., Raclet, J.B., Reinkemeier, P., Sangiovanni-Vincentelli, A., Damm, W., Henzinger, T.A., Larsen, K.G.: Contracts for System Design (2018)

6. Biere, A., Biere, A., Heule, M., van Maaren, H., Walsh, T.: Handbook of Satisfiability: Volume 185 Frontiers in Artificial Intelligence and Applications (2009)

7. Cavada, R., Cimatti, A., Dorigatti, M., Griggio, A., Mariotti, A., Micheli, A., Mover, S., Roveri, M., Tonetta, S.: The nuXmv Symbolic Model Checker. In: CAV. pp. 334-342 (2014)

8. Cimatti, A., Dorigatti, M., Tonetta, S.: OCRA: a tool for checking the refinement of temporal contracts. In: Proceedings of the 28th IEEE/ACM International Conference on Automated Software Engineering. pp. 702-705. ASE'13 (2013)

9. Dutertre, B.: Yices 2.2. In: Biere, A., Bloem, R. (eds.) Computer-Aided Verification (CAV'2014). Lecture Notes in Computer Science, vol. 8559, pp. 737-744 (July 2014)

10. Iannopollo, A., Tripakis, S., Sangiovanni-Vincentelli, A.L.: Constrained Synthesis from Component Libraries. In: FACS (2016)

11. Kellari, D., Crawley, E.F., Cameron, B.G.: Architectural Decisions in Commercial Aircraft from the DC-3 to the 787. Journal of Aircraft 55(2), 792-804 (2018)

12. Micouin, P.: Model-based systems engineering: fundamentals and methods. Control, systems and industrial engineering series, Hoboken, NJ (2014)

13. de Moura, L., Bjørner, N.: Z3: An Efficient SMT Solver. In: Tools and Algorithms for the Construction and Analysis of Systems. pp. 337-340. Berlin, Heidelberg (2008)

14. Nuzzo, P., Xu, H., Ozay, N., Finn, J.B., Sangiovanni-Vincentelli, A.L., Murray, R.M., Donzé, A., Seshia, S.A.: A Contract-Based Methodology for Aircraft Electric Power System Design. IEEE Access 2, 1-25 (2014)

15. Ribeiro dos Santos, C.A., Hany Saleh, A., Schrijvers, T., Nicolai, M.: CONDEnSe: Contract Based Design Synthesis. In: 2019 ACM/IEEE 22nd International Conference on Model Driven Engineering Languages and Systems (MODELS). pp. 250-260 (2019) 